\title{
Dimensional reduction for fast simulations of contact problems
}

\author{
T. Geike \& V. L. Popov \\ Institute of Mechanics, Technical University of Berlin, Germany
}

\begin{abstract}
This paper explains a method of how to reduce the dimension of a contact problem under study. In particular it is shown how the three-dimensional adhesive contact can be simulated on the basis of a one-dimensional model. Single contacts as well as multi-asperity contacts with adhesion are discussed in some detail. If lubricants are present it is necessary to solve simultaneously for elastic deformations of contacting bodies and fluid flow between the bodies. We show that the problem can be considerably reduced in the case when the lubrication layer is so thin that the main contribution to the contact interaction comes from a small part of micro contacts with a distance much smaller than the average distance between the bodies. In this case, it is possible to model the dynamics of lubrication by non-conservative forces between surface elements depending both on the distance and relative velocity. The presented reduction method is currently used for the simulation of chemical-mechanical polishing.

Keywords: elastic contacts, computational contact mechanics, friction, adhesion, lubrication, chemical-mechanical polishing.
\end{abstract}

\section{Introduction}

Contact and friction play an important role in many technical applications ranging from traditional applications like bearings, clutches and brakes [1] over manufacturing technologies $[2,3]$ to modern applications like micro-electromechanical systems [4]. Further technological progress in these fields requires a better understanding of the friction phenomenon and the development of appropriate simulation tools.

Many tribological systems belong to the class of fractal systems: in friction processes, both the microscopic and macroscopic scales may play an essential 
role $[4,5]$. The multi-scale nature of friction processes, however, makes the simulation of such systems difficult. The need of including many scales and physical processes in a simulation model leads to the development of reduced simulation methods.

One of the possibilities to reduce the computation time is to use hierarchical simulation methods [6]. In the present paper, another strategy is discussed: substitution of three-dimensional systems by one-dimensional systems. The basis of the reduction are the following two ideas [7]: (1) The elastic contact energy is a local quantity which only depends on the configuration and deformations in the vicinity of a micro contact, but does not depend on the size and the form of the body as a whole. (2) The interaction between micro contacts is of minor importance. Then the dimensionality of the multi-contact system plays no role as long as the behaviour of a single asperity and the statistics of asperities are modelled correctly.

The one-dimensional model is currently used to study the chemical-mechanical polishing [3]. In this application, elasticity, plasticity, lubrication, adhesion and the surface topography are considered in the simulation tool. Because of this complexity and the need to study a sufficiently huge piece of the specimen to be polished, simulating the evolution of the surface topography in the course of the polishing process is only possible with the proposed model.

In this paper the elastic contact is discussed in detail. Much attention is paid on how to set up the parameters of the one-dimensional model (elastic properties, surface topography). Subsequently two extensions are discussed: adhesion and lubrication.

\section{Elastic contact}

\subsection{Single contact}

The first main idea of the proposed reduced description is the following [7, 8]: consider the three-dimensional contact problem with relative radius of curvature $R_{3}$ and elastic modulus $E^{*}$. The relation between normal force $F_{3}$ and approach $d$ reads [9]

$$
F_{3}(d)=\frac{4}{3} E^{*} \sqrt{R_{3} d^{3}},
$$

while the relation between normal force $F_{3}$ and radius of contact $a$ is

$$
F_{3}(a)=\frac{4 E^{*}}{3 R_{3}} a^{3} .
$$

Now consider the one-dimensional contact problem, depicted in figure 1. The respective relations are

$$
F_{1}(d)=\frac{4 \sqrt{2} c_{n}}{3} \sqrt{R_{1} d^{3}},
$$




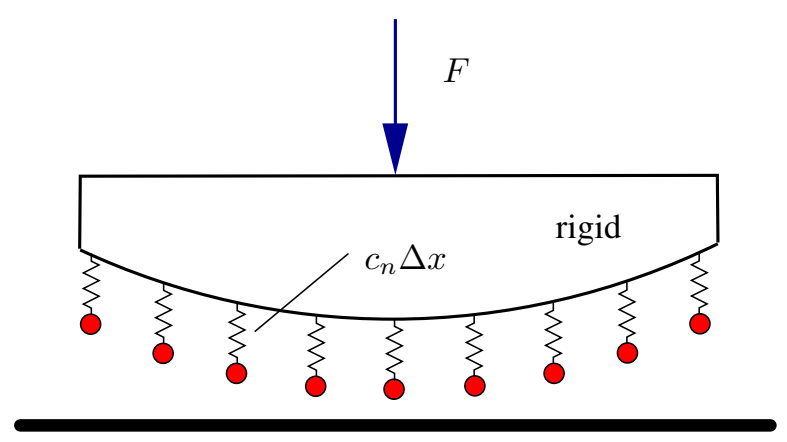

Figure 1: Contact between a rigid plate and a rigid cylinder with elastic layer.

$$
F_{1}(a)=\frac{2 c_{n}}{3 R_{1}} a^{3},
$$

where $c_{n}$ is the stiffness per unit length. Note that the distance $\Delta x$ between particles is small compared to the size of the contact. The macroscopic relations between force and approach and force and radius of contact will be identical for the three-dimensional and one-dimensional problem if

$$
R_{1}=\frac{1}{2} R_{3} \quad, \quad c_{n}=E^{*} .
$$

Hence, the three-dimensional contact problem can be reduced to a onedimensional problem for arbitrary radius of curvature. For the local force in the one-dimensional problem one gets

$$
f(x) \propto\left(a^{2}-x^{2}\right),
$$

which is different from the well-known result for the contact pressure in the threedimensional problem [9]. Introducing a stress according to

$$
\sigma(x)=\frac{f(x)}{b \sqrt{\delta(x) R_{1}}},
$$

where $\delta(x)$ is the local deformation and $b$ is the effective width, yields the desired relation

$$
\sigma(x) \propto \sqrt{1-\frac{x^{2}}{a^{2}}} .
$$

By choosing the effective width $b$ appropriately, the stress according to eqn (7) is identical to the three-dimensional result. When simulating problems with plasticity the yield criteria should depend on the stress $\sigma$ and not on the local force $f$.

\subsection{Multi-asperity contact}

The second important idea of the proposed 3D to 1D mapping is that the interaction between neighbouring asperities is of no importance for the contact problem as far 
as the size of micro contacts is much smaller than the distance between them - and this is the case in a typical macroscopic tribological system. It is rather the statistics of heights and radii of curvature which is important for the contact problem $[10,11]$. The statistics of micro contacts determines on one hand the normal forces between bodies. On the other hand, it determines the real area of contact and thus the tangential friction forces. The distribution of normal and tangential forces as well as the distribution of contact areas of micro contacts are the most important quantities for the understanding and the qualitative characterisation of tribological systems on the microscale. As we have shown that a single threedimensional asperity can be equivalently substituted by a one-dimensional asperity - independent of the radius of curvature - the next step is to create a onedimensional surface with the same statistical properties of the distributions of height and curvature as the two-dimensional surface of the three-dimensional body. It will then have the same contact properties as the inital three-dimensional body. In the present section we study the question whether it is possible to create such an equivalent one-dimensional surface (line) and if possible, how it has to be done correctly.

For simplicity, we assume here that a two dimensional surface topography (of the three-dimensional body) can be characterized by its surface roughness power spectra $C_{2 \mathrm{D}}(q)$ defined by

$$
C_{2 \mathrm{D}}(q)=\frac{1}{(2 \pi)^{2}} \int\langle h(\mathbf{x}) h(0)\rangle e^{-i \mathbf{q} \cdot \mathbf{x}} d^{2} x
$$

where $h(\mathbf{x})$ is the height measured from the average plane defined so that $\langle h\rangle=0$ and $\langle$.$\rangle stands for ensemble averaging. Since it is assumed that the statistical$ properties of the surface topography are translationally invariant and isotropic, the surface roughness power spectra $C_{2 \mathrm{D}}(q)$ only depends on the magnitude $q$ of the wave vector $\mathbf{q}[12-14]$.

Similarly a surface roughness power spectra $C_{1 \mathrm{D}}(q)$ can be introduced for a one-dimensional surface topography according to

$$
C_{1 \mathrm{D}}(q)=\frac{1}{2 \pi} \int\langle h(x) h(0)\rangle e^{-i q x} d x
$$

For generating a one-dimensional surface equivalent to the initial two-dimensional surface, the appropriate surface roughness power spectra $C_{1 \mathrm{D}}(q)$ must be defined. The qualitative arguments for the choice of the proper one-dimensional spectral density are the following: The height distribution of asperities of a fractal surface has generally the same order of magnitude as the mean square root value of the height $h(x)$ of the profile. The mean curvature of the asperities has the same order of magnitude as the mean square root value of the curvature $\kappa=\partial^{2} h(x) / \partial x^{2}$. 
The mean-square values of height for two- and one-dimensional systems

$$
\begin{aligned}
& \left\langle h^{2}\right\rangle_{2 \mathrm{D}}=2 \pi \int_{0}^{\infty} q C_{2 \mathrm{D}}(q) d q, \\
& \left\langle h^{2}\right\rangle_{1 \mathrm{D}}=2 \int_{0}^{\infty} C_{1 \mathrm{D}}(q) d q,
\end{aligned}
$$

will be equal, if we take

$$
C_{1 \mathrm{D}}(q)=\pi q C_{2 \mathrm{D}}(q) \quad .
$$

It is important to note, that the mean square curvatures $\left\langle\kappa^{2}\right\rangle$ will then be equal as well. We now show, that not only the average values of the heights and curvatures but also their distributions will be almost equal for these systems. Thus, the transformation eqn (13) defines the rule for generating equivalent onedimensional surfaces with the same contact properties as for three-dimensional bodies. For this sake, we study how the statistics of asperities of both two- and one-dimensional systems are related to each other. We generated one-dimensional and two-dimensional surface topographies and determined the statistics of their heights and curvatures numerically. The surface topography is calculated from the surface roughness power spectra according to

$$
h(\mathbf{x})=\sum_{\mathbf{q}} B_{2 \mathrm{D}}(\mathbf{q}) \exp (i(\mathbf{q} \cdot \mathbf{x}+\phi(\mathbf{q}))),
$$

where $\phi(\mathbf{q})=-\phi(-\mathbf{q})$ are randomly distributed in $[0,2 \pi)$ and

$$
B_{2 \mathrm{D}}(\mathbf{q})=\frac{2 \pi}{L} \sqrt{C_{2 \mathrm{D}}(\mathbf{q})}=\bar{B}_{2 \mathrm{D}}(-\mathbf{q}) .
$$

For the one-dimensional case the respective equations are

$$
\begin{aligned}
h(x) & =\sum_{q} B_{1 \mathrm{D}}(q) \exp (i(q x+\phi(q))), \\
B_{1 \mathrm{D}}(q) & =\sqrt{\frac{2 \pi}{L} C_{1 \mathrm{D}}(q)}=\bar{B}_{1 \mathrm{D}}(-q) .
\end{aligned}
$$

Numerical generation of surfaces is based on the FFT algorithm rather then on directly calculating the sums in eqns (14) and (15). For each generated surface topography the statistics of asperities is calculated. We introduce the following ratios $\phi_{1}, \phi_{2}$ and $\phi_{3}$ which relate the asperity statistics (index $p$ ) to the profile statistics.

$$
\phi_{1}=\sqrt{\frac{\left\langle h_{p}^{2}\right\rangle}{\left\langle h^{2}\right\rangle}}, \quad \phi_{2}=\frac{\left\langle\kappa_{p}\right\rangle}{\sqrt{\left\langle\kappa^{2}\right\rangle}}, \quad \phi_{3}=\sqrt{\frac{\left\langle\kappa_{p}^{2}\right\rangle}{\left\langle\kappa^{2}\right\rangle}} .
$$



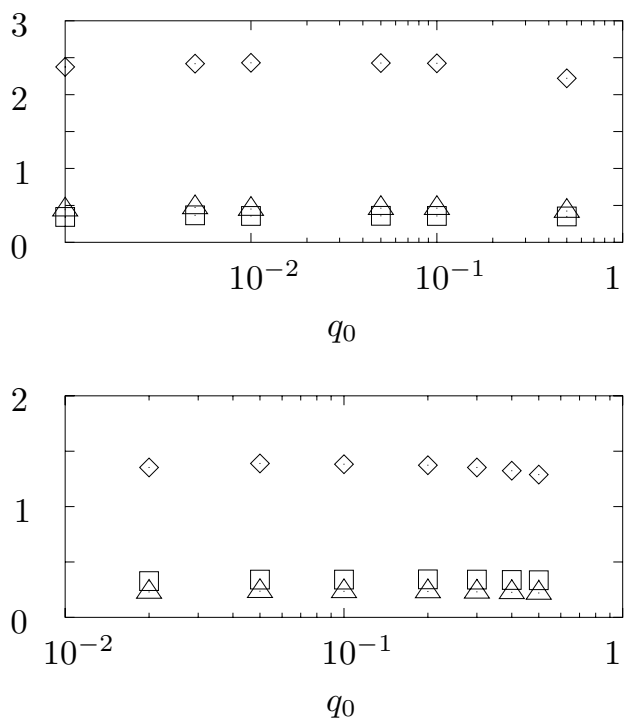

Figure 2: Ratios $\phi_{1}, \phi_{2}$ and $\phi_{3}$ according to eqn (17) for the 1D surface (top) and for the $2 \mathrm{D}$ surface (bottom), $q_{1}=2 q_{0}, \square \phi_{1}, \diamond \phi_{2}, \Delta \phi_{3}$.

Figure 2 shows $\phi_{1}, \phi_{2}$ and $\phi_{3}$ for one-dimensional surfaces (top) and for twodimensional surfaces (bottom), for a constant power spectrum with cutting wave vectors $q_{1}=2 q_{0}$

$$
C_{2 \mathrm{D}}=\left\{\begin{array}{ccc}
c & \text { for } & q_{0} \leq q \leq q_{1} \\
0 & & \text { otherwise }
\end{array}\right.
$$

and $C_{1 \mathrm{D}}$ according to eqn (13). $\phi_{1}, \phi_{2}$ and $\phi_{3}$ hardly depend on the wave number $q_{0}$. Further numerical experiments with $2 \leq q_{1} / q_{0} \leq 10$ show that this feature is also present for $q_{1} \neq 2 q_{0}$. From numerical studies with generated one- and two-dimensional surface topographies $\left(2 \leq q_{1} / q_{0} \leq 10\right)$ the following important conclusion regarding the statistics of asperities (index $p$ ) can finally be drawn: if the surface roughness power spectra are transformed according to eqn (13) the statistics of asperities will transform according to

$$
\begin{aligned}
& \left\langle h_{p}^{2}\right\rangle_{1 \mathrm{D}} \approx\left\langle h_{p}^{2}\right\rangle_{2 \mathrm{D}}, \\
& \left\langle\kappa_{p}\right\rangle_{1 \mathrm{D}} \approx 1.8\left\langle\kappa_{p}\right\rangle_{2 \mathrm{D}}, \\
& \left\langle\kappa_{p}^{2}\right\rangle_{1 \mathrm{D}} \approx 2.0\left\langle\kappa_{p}^{2}\right\rangle_{2 \mathrm{D}} .
\end{aligned}
$$

Note that according to eqn (5) the relation for the average curvature of asperties should preferably be $\left\langle\kappa_{p}\right\rangle_{1 \mathrm{D}}=2\left\langle\kappa_{p}\right\rangle_{2 \mathrm{D}}$. Choosing the stiffness $c_{n}$ to get the correct $F(d)$ relation, the contact radius $a$ will not be exactly equal in the two models. In the case at hand the relation $\left\langle\kappa_{p}\right\rangle_{1 \mathrm{D}} \approx 1.8\left\langle\kappa_{p}\right\rangle_{2 \mathrm{D}}$ leads to about $5 \%$ error in the radius of contact. 


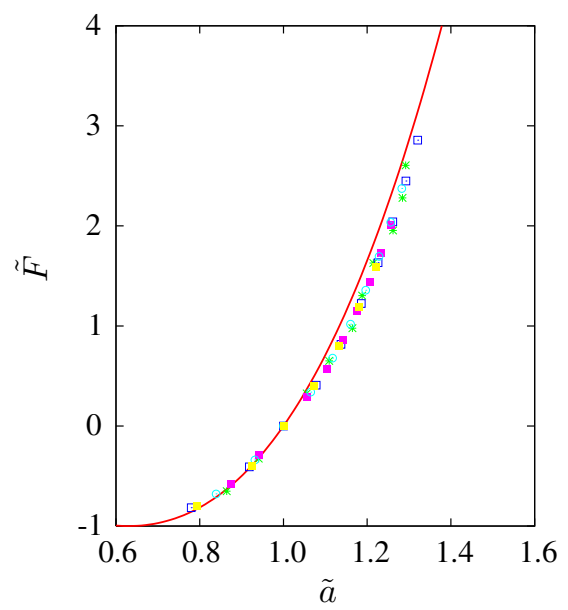

Figure 3: Non-dimensional normal force $\tilde{F}=F / F_{A}$ vs. non-dimensional contact size $\tilde{a}=a / a_{0}$, with adhesion force $F_{A}$ and contact radius $a_{0}$ at zero normal force, JKR result (solid line), simulation with the onedimensional model (points).

\section{Adhesion and lubrication}

\subsection{Adhesion}

For many contact and friction problems it is important to take adhesion into account. In particular this is important because adhesion may increase the real area of contact significantly even if no adhesion force is recognised in a pull-off experiment [15].

In the model discussed above adhesion is not considered, thus the interaction forces in this model (see figure 1) are repulsive forces only. Adhesion requires also attractive interaction forces. This can be done by using a Lennard-Jones-type interaction potential for the interactions between particles of opposing bodies.

Numerical experiments and analytical calculations with the one-dimensional model yield for the adhesion force $F_{A} \propto \sqrt{R_{1}}$. JKR theory [16] gives for the three-dimensional problem $F_{A} \propto R_{3}$. However for any given but fixed radius of curvature, the one-dimensional model gives the correct relation between normal force $F$ and radius of contact $a$. Figure 3 shows the JKR result (solid line) and results from numerical experiments with five different values for the radius of curvature. Thus the three-dimensional adhesive contact problem can be simulated on the basis of the proposed one-dimensional model under the restriction of having an arbitrary but fixed radius of curvature. Note that this restriction does not apply for the elastic contact without adhesion. 


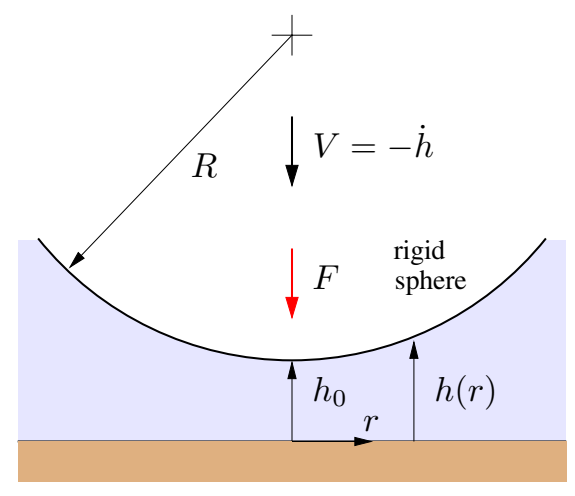

Figure 4: Normal approach of a rigid sphere.

Numerical results for the adhesive contact between rough surfaces especially the dependence of pull-off force on the roughness are presented in [17].

\subsection{Lubricated contacts}

The reduced description can also be extend to lubricated contacts under the condition that only asperities that are very close to asperities of the opposing body contribute significantly to the total force between the two macroscopic bodies. The reduction is based on the idea that mixed lubrication can be modelled by nonconservative forces between surface elements of the contacting bodies, instead of modelling fluid particles explicitly. Consider the normal approach of a rigid sphere and a rigid plate separated by a classical Newtonian fluid with constant viscosity $\eta$ (figure 4).

The normal force $F$ acting on the sphere can be calculated from the Reynolds equation and is

$$
F=-\frac{6 \pi \eta R^{2} \dot{h}}{h_{0}},
$$

where $-\dot{h}$ is the velocity of approach. Under the assumptions made the main contribution to the force comes from the immediate vicinity of the mirco contact. The details of the flow far away form the contact region do not influence the asperity-asperity interactions. The macroscopic result eqn (18) can actually be obtained in numerical simulations by introducing an interaction force

$$
F_{p p}=4.635 \frac{\eta R^{3 / 2} v}{r^{5 / 2}} d r_{1} d r_{2}
$$

where $r$ is the distance of the two interacting particles and $v$ is the projection of the relative velocity onto the direction between them. Note that the interaction force $F_{p p}$ between surface elements depends on the distance $r$ according to a simple power law and is proportional to the relative velocity. 
If cavitation is relevant in the application under study a reduced description is build on a kinetic equation for the interaction forces [18].

\section{Summary}

Starting from the idea that for many applications with contacts between randomly rough surfaces fast simulation tools are required we studied the problem whether it is possible to reduce the dimension of systems from three to one leaving the essential contact properties invariant. We have shown that it is indeed possible as long as the contact area is much smaller then the apparent (macroscopic) contact area. The reduction of the dimension means a huge reduction of computation time, allowing the simulation of multi-scale systems within one model.

The interaction forces between particles depend on the relative distance between particles and in the case of lubricated contacts on the relative velocity as well. Simulations of contacts between randomly rough surfaces also require a conversion of the surface roughness power spectra form two-dimensional to onedimensional.

A numerical implementation of the described model is currently used to study the chemical-mechanical polishing [3]. Extensive studies with three-dimensional and one-dimensional models are in progress that will give further information on the quality of the one-dimensional model.

\section{References}

[1] Ostermeyer, G.P. \& Müller, M., Dynamic interaction of friction and surface topography in brake systems. Tribology International, 39(5), pp. 370-380, 2006.

[2] Lovell, M.R. \& Deng, Z., Experimental investigation of sliding friction between hard and deformable surfaces with application to manufacturing processes. Wear, 236, pp. 117-127, 1999.

[3] Popov, V.L. \& Filippov, A.E., Modelling of mechanical polishing with lubrication. Technical Physics Letters, 31(9), pp. 788-792, 2005.

[4] Persson, B.N.J., Sliding Friction. Springer, 2nd edition, 2000.

[5] Persson, B.N.J., Bucher, F. \& Chiaia, B., Elastic contact between randomly rough surfaces: comparison of theory with numerical results. Physical Review B, 70(18), p. 184106, 2002.

[6] Yang, C., Tartaglino, U. \& Persson, B.N.J., A multiscale molecular dynamics approach to contact mechanics. European Physical Journal, 19(1), pp. 4758, 2006.

[7] Popov, V.L. \& Psakhie, S.G., Numerical simulation methods in tribology. Tribology International, 40(6), pp. 916-923, 2007.

[8] Geike, T. \& Popov, V.L., Reduction of three-dimensional contact problems to one-dimensional ones. Tribology International, 40(6), pp. 924-929, 2007. 
[9] Johnson, K.L., Contact mechanics. Cambridge University Press, 6th edition, 2001.

[10] Greenwood, J.A. \& Williamson, J.B., Contact of nominally flat surfaces. Proceedings of the Royal Society of London Series A, 295(1442), p. 300, 1966.

[11] Bush, A.W. \& Gibson, R.D., The elastic contact of a rough surface. Wear, 35, pp. 87-111, 1975.

[12] Persson, B.N.J., Elastic contact between randomly rough surfaces. Physical Review Letters, 87(11), p. 116101, 2001.

[13] Persson, B.N.J., Adhesion between elastic bodies with randomly rough surfaces. Physical Review Letters, 89(24), p. 245502, 2002.

[14] Palasantzas G. \& De Hosson J. Th. M., Influence of surface roughness on the adhesion of elastic films. Physical Review E, 67, p. 21604, 2003.

[15] Persson, B.N.J., Albohr, O., Tartaglino, U., Volokitin, A.I. \& Tosatti, E., On the nature of surface roughness with application to contact mechanics, sealing, rubber friction and adhesion. Journal of Physics: Condensed Matter, 17(1), pp. R1-R62, 2005.

[16] Johnson, K.L., Kendall, K. \& Roberts, A.D., Surface energy and contact of elastic solids. Proc Roy Soc London A, 324(1558), p. 301, 1971.

[17] Geike, T. \& Popov, V.L., Reduzierte Beschreibung des 3D adhäsiven Kontaktes zwischen rauen Oberflächen. Tribologie und Schmierungstechnik, 53(3), pp. 5-9, 2006.

[18] Geike, T. \& Popov, V.L., Reduced description of lubricated contacts with cavitation. Submitted to Tribology International. 